УДК $517.986+517.947$

DOI https://doi.org/10.24144/2616-7700.2020.2(37).130-141

\title{
M.I. Яременко
}

Національний технічний університет України "Київський політехнічний інститут імені Ігоря Сікорського" , Київ, доцент кафедри природничо-математичної освіти та інформаційних технологій, доцент, кандидат фізико-математичних наук

Math.kiev@gmail.com

ORCID: https://orcid.org/0000-0002-9209-6059

\section{КВАЗІЛІНІЙНІ СИСТЕМИ ПАРАБОЛІЧНИХ ДИФЕРЕНЦАЛЬНИХ РІВНЯНЬ В ДИВЕРГЕНТНІ ФОРМІ 3 ФОРМ-ОБМЕЖЕНИМИ КОЕФІЦІЕНТАМИ}

В роботі досліджуються квазілінійні системи параболічних диференціальних рівнянь в дивергентні формі другого порядку з сингулярними коефіцієнтами за умов форм-обмеженості і лінійного росту нелінійного збурення. Встановлюється існування розв'язку першої крайової задачі для квазілінійної системи параболічних диференціальних рівнянь за умов форм-обмеженості і лінійного росту в просторі Соболева. Розглядаються умови за яких нелінійне збурення параболічного диференціального оператору обмежене лінійною функцією з коефіцієнтами, які можуть бути сингулярними за просторовою зміною, в лінійному випадку ці коефіцієнти належать функціональним класам Като та Неша.

Ключові слова: квазілінійні системи, параболічні системи, простір Соболева, дивергентна форма, форм-обмеженість, сингулярні коефіцієнти, умови сингулярності.

1. Вступ. У всьому Евклідовому просторі $R^{l}, l \geq 3$ розглянемо квазілінійну систему параболічних диференціальних рівнянь в дивергентні формі

$$
\frac{\partial}{\partial t} \vec{u}-\sum_{i, j=1, \ldots, l} \frac{\partial}{\partial x_{i}}\left(a_{i j}(t, x, \vec{u}) \frac{\partial}{\partial x_{j}} \vec{u}\right)+\vec{b}(x, \vec{u}, \nabla \vec{u})=0, \quad l>2
$$

за умов строгої еліптичності, тобто, що виконується наступна нерівність

$$
\nu \sum_{i=1}^{l} \xi_{i}^{2} \leq \sum_{i j=1, \ldots, l} a_{i j} \xi_{i} \xi_{j} \leq \mu \sum_{i=1}^{l} \xi_{i}^{2} \quad \forall \xi \in R^{l}
$$

Подібні квазілінійні системи параболічних диференціальних рівнянь другого порядку вивчаються протягом довгого часу. Основні напрями досліджень регулярність розв'язків та існування розв'язку крайових задач були визначені 19-ою і 20-ою проблемами Гільберта [7], на розв'язання, яких були направлені зусилля багатьох видатних математиків. Зокрема, дослідження С.Н. Бернштейна, Ж. Лере [9], Ж. Ліонса [8], М.І. Вішика, Шаудера [9], Ш. Брезіса [18], А. Пазі [18, 21], Г. Мінті [33, 34], Ф. Браудера [19, 20], Де Джорджі, Д. Неша [38], Ю. Мозера [36], О.А. Ладиженської [9, 10], Н. Н. Уральцевої [9], Ж. Серріна, Трудінгера, Ю. А. Дубинського, С.І. Похожаєва, І.В. Скрипника [12], Н.В. Крилова $[1,6]$ і інших. У витоків створення основ теорї нелінійних еволюційних рівнянь стояли такі видатні математики, як I. Хілле, Р. Філліпс, К. Іосіда [4], М.I. Вішик, Т.Като [28-30], Й.Комура [31, 32], І. Міядера [35], Ж.Л. Ліонс [8], С.Г. Крейн, М.О. Красносельський [6], П.Є. Соболєвський, В. Барбю [13] і інші. 
В даній статті розробляється комбінація методу напівгруп та методу апріорних оцінок [46-50]. Для пояснення цього підходу розглянемо у всьому Евклідовому просторі $R^{l}, l \geq 3$ рівняння теплопровідності

$$
\partial_{t} u=\Delta u
$$

Фундаментальний розв'язок цього рівняння задається формулою

$$
p_{0}(t, x, y)=(4 \pi t)^{-\frac{l}{2}} \exp \left(-\frac{|x-y|^{2}}{4 t}\right), \quad t>0, \quad x, y \in R^{l} .
$$

Використовуючи фундаментальний розв'язок рівняння теплопровідності, можна дослідити більш складний варіант рівняння дифузії у наступному вигляді

$$
L u=\left[\frac{\partial}{\partial t}-\sum_{i, k=1, . ., l} a_{k j}(t, x) \nabla_{k} \nabla_{j}-\sum_{k=1, \ldots, l} b_{k}(t, x) \nabla_{k}\right] u(t, x)=0
$$

за умов $\exists \nu, \mu: 0<\nu \leq \mu<\infty$ такі, що

$$
\nu \sum_{i=1}^{l} \xi_{i}^{2} \leq \sum_{i j=1, \ldots, l} a_{i j}(t, x) \xi_{i} \xi_{j} \leq \mu \sum_{i=1}^{l} \xi_{i}^{2}
$$

і лінійне збурення $b_{k}(t, \cdot): R^{l} \mapsto R^{l}$.

Будемо використовувати наступні позначення

$$
\begin{gathered}
\nabla \circ a \circ \nabla u=\sum_{i, j=1, \ldots, l} \frac{\partial}{\partial x_{i}} a_{i j} \frac{\partial}{\partial x_{j}} u, \\
b \nabla u=b \circ \nabla u=\sum_{i=1, \ldots, l} b_{i} \frac{\partial}{\partial x_{i}} u .
\end{gathered}
$$

Розглянемо фундаментальний розв'язок

$$
p_{0}(t, x ; \tau, y)=(2 \pi)^{-l} \int \exp \left(i x \eta-\int_{\tau}^{t} a(\gamma, y) \eta^{2} d \gamma\right) d \eta
$$

параболічного рівняння

$$
\left[\partial_{t}-a_{k j}(t, y) \nabla_{k} \nabla_{j}\right] u(t, x)=0 .
$$

Можна показати, що

$$
\begin{gathered}
p_{0}(t, x ; \tau, y)=(2 \pi)^{-l} \int \exp \left(i x \eta-\int_{\tau}^{t} a(\gamma, y) \eta \cdot \eta d \gamma\right) d \eta= \\
=(2 \sqrt{\pi})^{-l}\left(\operatorname{det}\left(\int_{\tau}^{t} a(\gamma, y) d \gamma\right)\right)^{-\frac{1}{2}} \exp \left(\left(-\int_{\tau}^{t} a(\gamma, y) d \gamma\right)^{-1} \frac{(x, x)}{4}\right) .
\end{gathered}
$$

Наук. вісник Ужгород. ун-ту, 2020, вип. 37, № 2 ISSN 2616-7700 (print), 2708-9568 (online) 
З умов еліптичності маємо

$$
\begin{gathered}
\nu \sum_{i=1}^{l} \xi_{i}^{2}(t-\tau) \leq \int_{\tau}^{t} a(\gamma, y) d \gamma \eta \cdot \eta \leq \mu \sum_{i=1}^{l} \xi_{i}{ }^{2}(t-\tau), \\
\nu \sum_{i=1}^{l} \xi_{i}{ }^{2}(t-\tau)^{-1} \leq\left(\int_{\tau}^{t} a(\gamma, y) d \gamma\right)^{-1} \eta \cdot \eta \leq \mu \sum_{i=1}^{l} \xi_{i}{ }^{2}(t-\tau)^{-1},
\end{gathered}
$$

тоді одержуємо оцінку фундаментального розв'язку параболічного рівняння за допомогою Гаусової щільності

$$
\begin{gathered}
(2 \sqrt{\pi})^{-l} \nu^{\frac{-l}{2}}(t-\tau)^{\frac{-l}{2}} \exp \left(\frac{-\nu|x|^{2}}{4(t-\tau)}\right) \leq p_{0}(t, x ; \tau, y) \leq \\
\leq(2 \sqrt{\pi})^{-l} \mu^{\frac{l}{2}}(t-\tau)^{\frac{-l}{2}} \exp \left(\frac{-|x|^{2}}{4 \mu(t-\tau)}\right)
\end{gathered}
$$

Фундаментальний розв'язок рівняння (2) може бути представлений у вигляді

$$
p_{1}(t, x ; \tau, z)=p_{0}(t, x-z ; \tau, z)+\int_{\tau}^{t} d \eta \int p_{0}(t, x-y ; \eta, y) F(\eta, y ; \tau, z) d y
$$

де $F(\eta, y ; \tau, z)$ - щільність фундаментального розв'язку параболічного рівняння. Остання рівність може бути переписана у вигляді

$$
p_{1}(t, x ; \tau, z)=p_{0}(t, x-z ; \tau, z)+\int_{\tau}^{t} d \eta \int p_{0}(t, x-y ; \eta, y) b \circ \nabla p_{0}(\eta, y ; \tau, z) d y
$$

Оскільки клас $P K_{\beta}(A)$ складається з функцій $f \in L_{l o c}^{1}\left(R^{l}, d^{l} x\right)$ для яких виконується нерівність

$$
\left|\left\langle f|h|^{2}\right\rangle\right| \leq \beta\left\langle A^{\frac{1}{2}} h, A^{\frac{1}{2}} h\right\rangle+c(\beta)\|h\|_{2}^{2}
$$

для всіх гладких функцій $h \in D\left(A^{\frac{1}{2}}\right)$ тоді, якщо припустити, що $b \circ a^{-1} \circ b \in$ $P K_{\beta}(A)$ для деяких $\beta<1$, одержимо нерівність

$$
|\langle\nabla h \circ b h\rangle| \leq \sqrt{\beta}\langle A h, h\rangle+c(\beta) \frac{1}{2 \sqrt{\beta}}\|h\|_{2}^{2}, \quad h \in D\left(A^{\frac{1}{2}}\right)
$$

і у відповідності з КЛМН-теорем, існуе $C_{0}$ - напівгрупа $L^{\infty}$ - стиску $e^{-t \Lambda_{n}}, \frac{2}{2-\sqrt{\beta}} \leq$ $n \leq \infty$ така, що $\Lambda_{2}=A+b \circ \nabla$.

В частинному випадку, якщо $A \in$ оператором Лапласа отримуємо оцінку

$$
|\langle\nabla h \circ b h\rangle| \leq \sqrt{\beta}\|\nabla h\|^{2}+\frac{c(\beta)}{2 \beta}\|h\|^{2} \quad \forall h \in D(\Delta) .
$$

Розділ 1: Математика і статистика 
Теорема 1. Нехай

$$
\begin{gathered}
a(\cdot): \Omega \rightarrow R^{l} \otimes R^{l}, \quad a(\cdot) \in\left[L_{l o c}^{1}(\Omega)\right]^{l \times l}, \\
\nu \sum_{i=1}^{l} \xi_{i}{ }^{2} \leq \sum_{i j=1, \ldots, l} a_{i j}(t, x) \xi_{i} \xi_{j}, \quad \text { для деякux } \nu>0,
\end{gathered}
$$

при $q>\frac{l}{2}, \quad l>2 i$ збурення $b \cdot \nabla$ задовольняе умову

$$
b \circ a^{-1} \circ b \in L^{q}+L^{\infty} .
$$

Todi

1) Oператор $B_{1}=B_{1}(b)=\nabla \circ b$ визначений на області

$$
D\left(B_{1}\right)=\left\{u \in L^{1} ;|\nabla u| \in L_{l o c}^{1} ; b \circ \nabla u \in L^{1}\right\}
$$

$\epsilon A_{1}$ - обмеженим, тобто, $D\left(B_{1}\right) \supset D\left(A_{1}\right)$ i для всіх $\alpha>0, k(\alpha)<\infty$ виконуеться нерівність

$$
\left\|B_{1} h\right\|_{1} \leq \alpha\left\|A_{1} h\right\|_{1}+k(\alpha)\|h\|_{1}, \quad h \in D\left(A_{1}\right) .
$$

2) Існують числа $s>0$ i $\beta(s)<1$ такі, що

$$
\int_{0}^{s}\left\|B_{1} e^{-t A_{1}} h\right\|_{1} d t \leq \beta(s)\|h\|_{1}, \quad h \in D\left(A_{1}\right) .
$$

3) Oператор $A_{1}+B_{1}$ визначений на множині $D\left(A_{1}\right)$ породжує $C_{0}$ - напівгрупу $T_{1}^{t}$ сумісну з $T^{t}=\exp (-t(A+b \circ \nabla)) i$ для якоӥ є справедливою очінка

$$
\left\|T_{1}^{t}\right\|_{1 \rightarrow 1} \leq \frac{1}{1-\beta(s)} \exp \left(-t \frac{\log (1-\beta(s))}{s}\right), \quad t>0 .
$$

Контрприклад . Розглянемо лінійний оператор $-\Lambda_{p} \supset \nabla a \nabla-b \nabla$ з областю визначення $D\left(A_{p}\right)$, який породжує голоморфну напівгрупу в просторі $L^{p}\left(R^{l}, d^{l} x\right)$. Припустимо, що виконується умова $b \circ a^{-1} \circ b \in P K_{\beta}(A)$ і позначимо $b_{n}=\chi_{n} b$, де $b_{n}=\chi_{n} b$ індикаторна функція множини $\left\{x \in R^{l}: \quad\left(b \circ a^{-1} \circ b\right)(x) \leq n\right\}$, i існує рівномірна на $t \in[0,1]$ границя

$$
\text { strong } L^{p}-\lim _{n \rightarrow \infty} \exp \left(-t \Lambda_{p}\left(b_{n}\right)\right)=\exp \left(-t \Lambda_{p}(b)\right) \text {. }
$$

Тоді,

якщо $\beta<1, \quad p \in\left[\frac{2}{2-\sqrt{\beta}}, \infty\left[\right.\right.$ то оператор $A+b \nabla$ породжує $C_{0^{-}}$напівгрупу стиску, для якої виконуються нерівності

$$
\begin{gathered}
\left\|\exp \left(-t \Lambda_{p}\right)\right\|_{p \rightarrow p} \leq \exp \left(\frac{c(\beta) t}{p-1}\right) \\
\left\|\exp \left(-t \Lambda_{p}\right)\right\|_{p \rightarrow s} \leq C \exp \left(\frac{c(\beta) t}{\sqrt{\beta}}\right) t^{\frac{-(s-p) l}{2 p s}}, \frac{2}{2-\sqrt{\beta}}<p<s \leq \infty ;
\end{gathered}
$$

Наук. вісник Ужгород. ун-ту, 2020, вип. 37, № 2 ISSN 2616-7700 (print), 2708-9568 (online) 
якщо $1 \leq \beta<4, \quad p<s \in\left[\frac{2}{2-\sqrt{\beta}}, \infty[\right.$ то операторна сума $A+b \nabla$ визначена некоректно, але напівгрупа існує і може бути заданою у вигляді границі

$$
\exp \left(-t \Lambda_{p}(b)\right) \equiv \text { strong } L^{p}-\lim _{n \rightarrow \infty} \exp \left(-t \Lambda_{p}\left(b_{n}\right)\right), \quad t \geq 0,
$$

дана границя є означенням напівгрупи.

Основною метою даної роботи є встановлення умов щодо нелінійності за яких перша крайова задача для даної системи (1) буде мати принаймні один розв'язок.

2. Постановка задачі та оцінка енергетичного типу. Розглянемо параболічну систему

$$
\frac{\partial}{\partial x} u^{k}-M(\vec{u})=0, \quad k=1, \ldots, N, \quad l>2,
$$

де позначено нелінійний диференціальний векторний оператор, вигляду:

$$
M(\vec{u})=\sum_{i, j=1, \ldots, l} \frac{\partial}{\partial x_{i}}\left(a_{i j}(t, x, \vec{u}) \frac{\partial}{\partial x_{j}} u^{k}\right)-b^{k}(x, \vec{u}, \nabla \vec{u}) \quad k=1, \ldots, N, \quad l>2,
$$

за умов: для довільного елементу $\vec{u} \in W_{1}^{p}\left(R^{l}, d^{l} x\right), l>2$ існують такі постійні додатні величини $v(\vec{u}), \mu(\vec{u})$, що виконується наступні нерівності

1. $v(\vec{u}) \sum_{i=1}^{l} \xi_{i}^{2} \leq \sum_{i j=1, \ldots, l} a_{i j}(t, x, \vec{u}) \xi_{i} \xi_{j} \leq \mu(\vec{u}) \sum_{i=1}^{l} \forall \xi \in R^{l}$

2. $\left|a_{i j}(t, x, \vec{u}) \xi_{i} \xi_{j}-a_{i j}(t, x, \vec{v}) \xi_{i} \xi_{j}\right| \leq \mu_{6} \sum_{i=1}^{l} \xi_{i}^{2} \quad \forall \xi \in R^{l}$

3. $b(t, x, y, z) \in$ вимірною векторною функцією своїх аргументів і $b \in L_{l o c}^{l} R^{l}$;

4. вектор-функція $b(t, x, y, z)$ майже скрізь задовольняє нерівності:

$$
|b(t, x, u, \nabla u)| \leq \mu_{1}(x)|\nabla u|+\mu_{2}(x)|u|+\mu_{3}(x),
$$

де $\mu_{1}^{2} \in P K_{\beta}(A), \mu_{2} \in P K_{\beta}(A)$, функція $\mu_{3} \in L^{p}\left(R^{l}\right)$;

5. приріст вектор-функції $b(x, y, z)$ майже скрізь задовольняє умову:

$$
|b(t, x, u, \nabla u)-b(t, x, v, \nabla v)| \leq \mu_{4}(x)|\nabla(u-v)|+\mu_{5}(x)|u-v|,
$$

де $\mu_{4}^{2} \in P K_{\beta}(A), \mu_{5} \in P K_{\beta}(A)$.

Побудуємо форму $h:\left(\begin{array}{l}N \\ \times \\ 1\end{array} W_{1}^{p}\left(R^{l}, d^{l} x\right)\right) \times\left(\begin{array}{l}N \\ \times \\ 1\end{array} W_{1}^{q}\left(R^{l}, d^{l} x\right)\right) \rightarrow R$ :

$$
\begin{aligned}
h(u, v) & \left.\equiv\langle u(t, u), v(t, u)\rangle\right|_{0} ^{T}-\int_{0}^{T}\left\langle u(t, u), \frac{\partial}{\partial t} v(t, u)\right\rangle+ \\
& +\int_{0}^{T}\left\langle\sum_{i, j=1, \ldots, l} a_{i j} \frac{\partial}{\partial x_{j}} u, \frac{\partial}{\partial x_{i}} v\right\rangle d t+\int_{0}^{T}\langle b, v\rangle d t,
\end{aligned}
$$

Розділ 1: Математика і статистика 
яку будемо вважати визначеною для всіх елементів $u \in W_{1}^{p}\left(R^{l}, d^{l} x\right)$ та $v \in$ $W_{1}^{q}\left(R^{l}, d^{l} x\right)$.

Якщо функцію $v$ вибрати $v(t)=u(t)|u(t)|^{p-2}, \partial_{t} v=(p-1)|u|^{p-2} \partial_{t} u$, тоді отримаємо

$$
\begin{gathered}
h\left(u, u|u|^{p-2}\right)=\left.\|u\|_{p}^{p}\right|_{0} ^{T}+ \\
\int_{0}^{T}\left(-(p-1)\left\langle u,|u|^{p-2} \partial_{t} u\right\rangle+\left\langle\sum_{i, j=1, \ldots, l} a_{i j} \frac{\partial}{\partial x_{j}} u, \frac{\partial}{\partial x_{i}}\left(u|u|^{p-2}\right)\right\rangle\right) d t+ \\
+\int_{0}^{T}\left\langle b, u|u|^{p-2}\right\rangle d t \\
h\left(u, u|u|^{p-2}\right)=\left.\frac{1}{p}\|u\|_{p}^{p}\right|_{0} ^{T}+4 \frac{p-1}{p^{2}} \int_{0}^{T}\left\langle\sum_{i, j=1, \ldots, l} a_{i j} \frac{\partial}{\partial x_{j}}\left(u|u|^{\frac{p-2}{2}}\right), \frac{\partial}{\partial x_{i}}\left(u|u|^{\frac{p-2}{2}}\right)\right\rangle d t+ \\
+\int_{0}^{T}\left\langle b, u|u|^{p-2}\right\rangle d t
\end{gathered}
$$

Покладемо $\left\|u|u|^{p-2}\right\|^{q}=\left\langle|u|^{(p-1) q}\right\rangle=\|u\|^{p}=\|w\|^{2}$, тоді отримаємо оцінку енергетичного типу

$$
\begin{gathered}
\left|h\left(u, u|u|^{p-2}\right)\right| \leq\left.\frac{1}{p}\|w\|^{2}\right|_{0} ^{T}+4 \frac{p-1}{p^{2}} \int_{0}^{T}\langle\nabla w \circ a \circ \nabla w\rangle d \tau+ \\
+\left(\frac{1}{q \sigma^{q}}+\frac{c(\beta)}{p} \epsilon^{2}+c(\beta)+\frac{1}{q \gamma^{q}}\right) \int_{0}^{T}\|w\|^{2} d t+ \\
+\left(\frac{1}{p}\left(\frac{1}{\epsilon^{2}}+\beta \epsilon^{2}\right)+\beta\right) \int_{0}^{T}\langle\nabla w \circ a \circ \nabla w\rangle d t+\frac{\gamma^{p}}{p} \int_{0}^{T}\left\|\mu_{3}\right\|^{p} d t .
\end{gathered}
$$

3. Перша крайова задача. Розглянемо першу крайову задачу для квазілінійної системи параболічних диференціальних рівнянь, у вигляді

$$
\frac{\partial}{\partial t} u^{k}-\sum_{i, j=1, \ldots, l} \frac{\partial}{\partial x_{i}}\left(a_{i j}(t, x, \vec{u}) \frac{\partial}{\partial x_{j}} u^{k}\right)+b^{k}(x, \vec{u}, \nabla \vec{u})=0, \quad k=1, \ldots, N, \quad l>2
$$

з граничними умовами

$$
\vec{u}(S(T))=0, \vec{u}(0, x)=\vec{\varphi}(x)
$$

Доведемо існування розв'язку цієї задачі у функціональному просторі $V_{1}^{2}$. Для цього припустимо, що $\left\{\vec{v}_{k}(x)\right\}, \quad k=1,2, \ldots$, є ортогональним базисом в просторі $W_{1,0}^{m}\left(R^{l}, d^{l} x\right), l>2$, таким, що $\left\langle\vec{v}_{k}, \vec{v}_{r}\right\rangle=\delta_{k r}$ i $\max \left|\vec{v}_{k}, \nabla \vec{v}_{k}\right| \leq c_{k}<\infty$. Наближений розв'язок $\vec{u}_{n}(t, x)$ будемо шукати у вигляді $\vec{u}_{n}=\sum_{i=1, \ldots, n} \vec{c}_{i}^{n}(t) \vec{v}_{i}(x)$, де коефіцієнти $\vec{c}_{i}^{n}(t)$ визначаються з системи звичайних диференціальних рівнянь

$$
\left\langle\partial_{t} \vec{u}_{n}, \vec{v}_{r}\right\rangle+\left\langle\sum_{i, j=1, \ldots, l} a_{i j} \frac{\partial}{\partial x_{j}} \vec{u}_{n}, \frac{\partial}{\partial x_{i}} \vec{v}_{r}\right\rangle+\left\langle\vec{b}, \vec{v}_{r}\right\rangle=0, \quad r=1, \ldots, n,
$$

і початкових умов

$$
\vec{c}_{i}^{n}(0)=\left\langle\vec{\varphi}, \vec{v}_{i}\right\rangle, \quad i=1, \ldots, n
$$

Наук. вісник Ужгород. ун-ту, 2020, вип. 37, № 2 ISSN 2616-7700 (print), 2708-9568 (online) 
Оскільки другий і третій доданки є вимірними і обмеженими на всіх множинах $\left\{t \in[0, T], \quad\left|\vec{c}_{i}^{n}\right| \leq\right.$ const $\}$ функціями від $t$, тому, якщо всі можливі розв'язки рівномірно обмежені на $[0, T]$ то на інтервалі $[0, T]$ існує розв'язок $\vec{c}_{i}^{n}(t)$, який задовольняе початкову умову $\vec{c}_{i}^{n}(0)=\left\langle\vec{\varphi}, \vec{v}_{i}\right\rangle, i=1, \ldots, n$.

Функції $\left\langle\vec{u}_{n}, \vec{v}_{i}\right\rangle, \quad n, i=1, \ldots, \in$ неперервними по $t \in[0, T]$. Потрібно показати, що функції $\left\langle\vec{u}_{n}, \vec{v}_{i}\right\rangle, \quad n \in$ рівностепенно неперервні по $t \in[0, T]$ для всіх фіксованих $i$.

Якщо всі можливі розв'язки рівномірно обмежені на $[0, T]$ і функції $\left\langle\vec{u}_{n}, \vec{v}_{i}\right\rangle$, $n \in \mathbb{N} \in$ рівностепенно неперервні по $t \in[0, T]$ для всіх фіксованих $i$, тоді із послідовності розв'язків $\vec{u}_{n}(t, x)$ можна виділити підпослідовність $\vec{u}_{n(s)}$ таку, що підпослідовність $\frac{\partial}{\partial x_{j}} \vec{u}_{n(s)}(t, x)$ збігається до $\frac{\partial}{\partial x_{j}} \vec{u}(t, x)$ слабко в просторі Лебега. Позначимо цю підпослідовність $\vec{u}_{n(s)}$ через $\vec{u}_{n}$, тобто, будемо вважати, що початкова послідовність співпадає з підпослідовністю.

Встановимо апріорну оцінку розв'язків на $[0, T]$, для цього помножимо

$$
\int_{0}^{T}\left\langle\partial_{t} \vec{u}_{n}, \vec{v}_{r}\right\rangle d t+\int_{0}^{T}\left\langle\sum_{i, j=1, \ldots, l} a_{i j} \frac{\partial}{\partial x_{j}} \vec{u}_{n}, \frac{\partial}{\partial x_{i}} \vec{v}_{r}\right\rangle d t+\int_{0}^{T}\left\langle\vec{b}, \vec{v}_{r}\right\rangle d t=0
$$

де $r=1, \ldots, n$, на $\vec{c}_{r}^{n}$ і просумуємо по $r$ від 1 до $n$, одержимо

$$
\frac{1}{2}\left\|\vec{u}_{n}\right\|_{2}^{2}+\int_{0}^{T}\left\langle\sum_{i, j=1, \ldots, l} a_{i j} \frac{\partial}{\partial x_{j}} \vec{u}_{n}, \frac{\partial}{\partial x_{i}} \vec{u}_{n r}\right\rangle d t+\int_{0}^{T}\left\langle\vec{b}, \vec{u}_{n}\right\rangle d t=0, \quad r=1, \ldots, n
$$

оцінюемо

$$
\begin{gathered}
\left.\frac{1}{2}\left\|\vec{u}_{n}\right\|_{2}^{2}\right|_{0} ^{T}+\nu \int_{0}^{T}\left\|\nabla \vec{u}_{n}\right\|_{2}^{2} d t \leq\left(\frac{1}{2 \sigma^{2}}+\frac{c(\beta)}{2} \varepsilon^{2}+c(\beta)+\frac{1}{2 \gamma^{2}}\right) \int_{0}^{T}\left\|\vec{u}_{n}\right\|_{2}^{2} d t+ \\
+\left(\frac{1}{2}\left(\frac{1}{\varepsilon^{2}}+\beta \varepsilon^{2}\right)+\beta\right) \int_{0}^{T}\left\|\nabla \vec{u}_{n}\right\|_{2}^{2} d t+\frac{\gamma^{2}}{2} \int_{0}^{T}\left\|\mu_{3}\right\|^{2} d t .
\end{gathered}
$$

З останньої нерівності випливає апріорна оцінка послідовності розв'язків $\vec{u}_{n}(t, x)$. Покажемо, що $\vec{u}(t, x)$ є розв'язком, для довільної функції $w=\sum_{i=1, \ldots, n} d_{i}(t) \vec{v}_{i}(x)$, де $d_{i}(t)$ неперервні функції узагальнені похідні яких є обмеженими на інтервалі $[0, T]$. Множину таких функцій $\vec{w}=\sum_{i=1, \ldots, n} \vec{d}_{i}(t) \vec{v}_{i}(x)$ позначимо $\wp(n)$.

Складемо інтегральні тотожності

$$
\begin{gathered}
-\int_{0}^{t}\left\langle\vec{u}_{n}, \partial_{t} \vec{w}\right\rangle d t+\left.\left\langle\vec{u}_{n}, \vec{w}\right\rangle\right|_{0} ^{t}+\int_{0}^{t}\left\langle\sum_{i, j=1, \ldots, l} a_{i j} \frac{\partial}{\partial x_{j}} \vec{u}_{n}, \frac{\partial}{\partial x_{i}} \vec{w}\right\rangle d t+\int_{0}^{t}\langle\vec{b}, \vec{w}\rangle d t=0, \\
t \in[0, T] .
\end{gathered}
$$

Оскільки функція $\vec{u}_{n}(t, x)$ належить множині $\wp(n) \quad \forall n \in \mathrm{N}$ то перейдемо до границі при $n \rightarrow \infty$, одержимо

$$
-\int_{0}^{t}\left\langle\vec{u}, \partial_{t} \vec{w}\right\rangle d t+\left.\langle\vec{u}, \vec{w}\rangle\right|_{0} ^{t}+\int_{0}^{t}\left\langle\sum_{i, j=1, \ldots, l} a_{i j} \frac{\partial}{\partial x_{j}} \vec{u}, \frac{\partial}{\partial x_{i}} \vec{w}\right\rangle d t+\int_{0}^{t}\langle\vec{b}, \vec{w}\rangle d t=0,
$$

Розділ 1: Математика і статистика 
де $t \in[0, T]$. Ця тотожність справедлива для довільної функції $w \in \bigcup_{n \in \mathrm{N}} \wp(n)$.

Отже, функція $\vec{u}(t, x) \in$ розв'язком першої крайової задачі. Залишилося довести, що функцій $\left\langle\vec{u}_{n}, \vec{v}_{i}\right\rangle, n \in \mathrm{N} \in$ рівностепенно неперервні по $t \in[0, T]$ для всіх фіксованих $i$, дійсно, одержуємо

$$
\begin{gathered}
\left|\left\langle\vec{u}_{n}(t+\Delta t, x)-\vec{u}_{n}(t, x), \vec{v}_{r}\right\rangle\right| \leq \int_{t}^{t+\Delta t}\left|\left\langle\sum_{i, j=1, \ldots, l} a_{i j} \frac{\partial}{\partial x_{j}} \vec{u}_{n}, \frac{\partial}{\partial x_{i}} \vec{v}_{r}\right\rangle\right| d t+ \\
+\int_{t}^{t+\Delta t}\left|\left\langle\vec{b}, \vec{v}_{r}\right\rangle\right| d t \leq\left(\frac{1}{2 \sigma^{2}}+\frac{c(\beta)}{2} \varepsilon^{2}+c(\beta)+\frac{1}{2 \gamma^{2}}\right) \int_{t}^{t+\Delta t}\left\|\vec{u}_{n}\right\|_{2}^{2} d t+ \\
+\left(\frac{1}{2}\left(\frac{1}{\varepsilon^{2}}+\beta \varepsilon^{2}\right)+\beta+\mu\right) \int_{t}^{t+\Delta t}\left\|\nabla \vec{u}_{n}\right\|_{2}^{2} d t+\frac{\gamma^{2}}{2} \int_{t}^{t+\Delta t}\left\|\mu_{3}\right\|^{2} d t . \\
\leq \text { const } \cdot \Delta t \stackrel{\Delta t \rightarrow 0}{\longrightarrow} 0
\end{gathered}
$$

що доводить рівностепенну неперервність функцій $\left\langle\vec{u}_{n}, \vec{v}_{i}\right\rangle, \quad n \in \mathrm{N}$.

Отже, доведено теорему 1.

Теорема 1. Нехай виконуються умови 1-5. Тоді перша крайова задача для системи (3) для довільної функиї $\vec{\varphi}(x) \in L^{2}$ мае принаймні один розв'язок в просторі $V_{1}^{2}$ при $t \in[0, T]$ i виконуеться умова

$$
\lim _{\Delta t \rightarrow 0} \frac{\|u(t+\Delta t, x)-u(t, x)\|_{2}^{2}}{\Delta t}=0 .
$$

4. Висновки та перспективи подальших досліджень. Встановлено, що перша крайова задача для квазілінійної системи параболічних диференціальних рівнянь другого порядку за умов форм-обмеженості і лінійного росту має розв'язок у просторі Соболева. В наступних умовах дані планується розширити клас систем, які можуть бути досліджені за допомогою даного методу.

\section{Список використаної літератури}

1. Ахиезер Н.И. Теория линейных операторов в гильбертовом пространстве. М.: Гостехиздат, 1950. $543 \mathrm{c.}$

2. Самойленко А.М., Бігун Я.Й. Усереднення нелінійних коливних систем вищого наближення із запізненням. Нелінійні коливання. 2002. Т. 5, № 1. С. $77-85$.

3. Бойчук И.А. Нелинейная нетерова краевая задача в критическом случае. Доповіді НАН України. 2010. № 3. С. $35-40$.

4. Иосида К. Функциональный анализ. М.: Мир, 1967. 624 с.

5. Коваленко В.Ф., Кухарчук Н.М., Семенов Ю.А. К теории диффузионных процессов, порождаемых оператором $\frac{1}{2} \Delta+d \nabla$. Деп. в УкрНИИНТИ. Киев, 1985. №2380-Ук 85.

6. Красносельский М.А. Положительные решения операторных уравнений. М.: Гос. изд-во физ.-мат. лит-ры. 1962. 394c.

7. Ладыженская О.А., Солонников В.А., Уральцева Н.Н. Линейные и квазилинейные уравнения параболического типа. М.: Наука, 1967. 735 с.

8. Лионс Ж.-Л. Некоторые методы решения нелинейных краевых задач. М: Мир, 1972. 587 c.

9. Ладыженская О.А., Уральцева Н.Н. Линейные и квазилинейные уравнения эллиптического типа. М.: Наука, 1973. 579 с.

10. Олейник О.А., Самохин В.Н. Математические методы в теории пограничного слоя. М.: Физматлит, 1997. 512 с.

11. Семенов Ю.А. Гладкость обобщенных решений уравнения $\left(\lambda-\sum_{i, j} \nabla_{i} a_{i j} \nabla_{j}\right) u=f$ с непрерывными коэффициентами. Мат. сб. 1982. Т.118 (160), №3 (7). С. 399 - 410. 
12. Скрипник И.В. Необходимое условие регулярности граничной точки для квазилинейного параболического уравнения. Матем. сб. 1992. Т.183, №7. С. 3-22.

13. Barbu V. Nonlinear semigroups and differential equations in Banach spaces. Legden: Nordhoff International Publiching, 1976. 352 p.

14. Benjamini I., Chavel I., Feldman E.A. Heat kernel lower bounds on Riemannian manifolds using the old ideas of Nash. Proc. London Math. Soc. 1996. V.72. P. $215-240$.

15. Belluce L.P., Kirk W.A. Fixed point theorems for families of contraction mappings. Pacific J. Math. 1966. V.18, № 2. P. 213 - 217.

16. Berlyiand A.G., Semenov Yu. A. On the $L_{p}$-theory of Schrodinger semigroups. Siberian Math. J. 1990. V.31. P. $16-26$.

17. Boychuk I. Weakly perturbed nonlinear boundary-value problem in critical case. Studies of the University of Žilina. Mathematical Series. October, 2009. V. 23, № 1. P. 1-8.

18. Brézis H., Pazy A. Semigroups of non-linear contractions on convex sets. J. Func. Anal. 1970. V. 6. P. 237-281.

19. Browder F.E. Existence of periodic solutions for nonlinear equations of evolution. Proc. Nat. Acad. Sci. USA. 1965. V. 53. P. 1100 - 1103.

20. Browder F.E. Nonlinear equations of evolution type and nonlinear accretive operators in Banach spaces. Bull. Amer. Math. Soc. 1967. V.73. P. 867 - 874.

21. Crandall M.G., Pazy A. Nonlinear semi-groups of contractions and dissipative sets. J. Func. Anal. 1969. V. 3. P. $376-418$.

22. Chichurin A.V. Integration of Chazy equation with constant coefficients. Nonlinear Oscillations. 2003. Vol. 6, № 1. P. 133-143.

23. Chichurin A.V. Integration of special linear equations of the second order. Nonlinear Oscillations. 2003. Vol. 6, № 2. P. 279-287.

24. David E.E., Evans W.D. Hardy operators, functional spaces and embeddings. Berlin: Springer, 2004. 326 p.

25. Fabes E.B. Gaussian upper bounds on fundamental solutions of the parabolic equation: the method of Nash in Dirichlet forms. Lectures Notes in Math. Berlin: Springer-Verlag, 1993. P.1 -20 .

26. Goldstein J. Semigroups of linear operators and applications. Oxford: Oxford University Press, 1985. $245 \mathrm{p}$.

27. Kasyanov P., Zadoyanchuk N. Faedo-Galerkin method for the second-order nonlinear evolution equations with the operators of the Volterra type. International Conference on Differential Equations Dedicated to the 100th Anniversary of Ya.B.Lopatynsky: Book of Abstracts (Lviv, September 12-17, 2006)/Ivan Franko National University of Lviv. Lviv, Ivan Franko National University of Lviv, 2006. P.104-105.

28. Kato T. Nonlinear semigroups and evolution equations. J. Math. Soc. Japan. 1967. V. 3. P. $375-402$.

29. Kato T. Perturbation theory for linear operators. Berlin-Heidelberg-New York: SpringerVerlag, 1980. $578 \mathrm{p}$.

30. Kato T. Non-linear semigroups and evolution equations. J. Math. Soc. Japan. 1967. V. 19. P. $508-520$.

31. Komura Y. Differentiability of nonlinear semigroups. J. Math. Soc. Japan. 1969. V. 21. P. $375-402$.

32. Komura Y. Nonlinear semi-groups in Hilbert space. J. Math. Soc. Japan. 1967. V. 19. P. 493 $-507$.

33. Minty G. Monotone (nonlinear) operators in Hilbert space. Duke Math. J. 1962. V. 29. P. 341 -346 .

34. Minty G. On the generalization of a direct method of the calculus of variations. Bull. Amer. Math. Soc. 1967. V. 73, №3. P. 315 - 321.

35. Miyadera I. On perturbation theory for semi-groups of operators. Tohoku Math. J. 1966. V. 18. P. $299-310$.

36. J. Moser, A new proof of De Giorgi's theorem concerning the regularity problem for elliptic differential equations, Comm. Pure Appl. Math. 13 1960. P. 457-468.

37. Nagy B. Spectral mapping theorems for semigroups of operators. Acta Science Math. 1976. V. 38. P. 343-351.

Розділ 1: Математика і статистика 
38. Nash J. Continuity of solutions of parabolic and elliptic equations. Amer. J. Math. 1958. V. 80. P. $931-954$.

39. Naniewicz Z.,Panagiotopoulos P.D. Mathematical theory hemivariational inequalities and applications. Marcel Dekker, Inc., New York, Basel Hong Kong. 1995. 267 p.

40. Nirenberg L. Remarks on strongly elliptic partial differential equations. Comm. Pure Appl. Math. 1955. V. 8. P. 648-674.

41. Opial Z. Weak convergence of the sequences of successive approximants for non-expansive mappings in Banach spaces. Bull. Amer. Math. Soc. 1967. V. 73. P. 591 - 597.

42. Pederson R.N. On an inequality of Opial, Beesack and Levinson. Proc. Amer. Math. Soc. 1965. V. 16. P.174 - 234.

43. Papageorgiou N.S. Existence of solutions for the second order evolution inclusion. Journal of applied mathematics and stochastic analysis. 1994. Vol.7, № 4. P. 525-535.

44. Papageorgiou N.S. Second order nonlinear evolution inclusions: structure of the solution set. Acta math. sinica, English series. 2006. Vol. 22 № 1. P. 195-206.

45. Papageorgiou N.S. On multivalued evolutions equations and differential inclusions in Banach spaces. Comment. math. unaiv. San. Pauli. 1987. Vol. 36. P. 21-39.

46. Yaremenko M.I. Second order quisi-linear elliptic equation with matrix of Gilbarg - Serrin in $R^{l}$ and nonlinear semi-groups of contraction in $L^{p}$. Матеріали конференції «Дванадцята міжнародна наукова конференція імені академіка М. Кравчука. 15-17 травня 2008 року, Київ». Київ, 2008. С. 473.

47. Yaremenko M.I. Second order quisi-linear elliptic equation with matrix of Gilbarg - Serrin in $R^{l}$ and nonlinear semi- groups of contraction in $L^{p}$. Матеріали конференції «nternational Conference on problems of decision making under uncertainties (PDMU-2008). May 12-17, 2008.» 2008. C.43.

48. Yaremenko M.I. The existence of solution of evolution and elliptic equations with singular coefficients. Asian Journal of Mathematics and Computer Research. 2017. Vol.: 15, Issue.: 3. pp. 172- 204.

49. Yaremenko M.I. Quasi-linear evolution and elliptic equations. Journal of Progressive Research in Mathematics. Vol.11., №3. 2017 pp. 1645-1669.

50. Yaremenko M.I. Sequence of semigroups of nonlinear operators and their applications to study the Cauchy problem for parabolic equations. Scientific Journal of the ternopil national technical university № 4 (84). 2016. pp. 149-160.

Yaremenko M. I. Quasilinear system of parabolic differential equations in the divergent form under form-boundary conditions.

In this article we study quasilinear systems of parabolic differential equations in divergent forms of the second order with the singular coefficients under conditions of formboundedness and linear growth of nonlinear perturbation. The existence of a solution of the first boundary value problem for a quasilinear system of parabolic differential equations under conditions of bounded forms and linear growth in Sobolev space is established. We consider the conditions under which nonlinear perturbation is bounded by a linear function with coefficients that can be spatially singular, in the linear case these coefficients belong to the Kato and Nash functional classes.

Ключові слова: quasilinear system, parabolic system, Sobolev space, divergent form, form-boundedness, singular coefficient, singularity.

\section{References}

1. Ahiezer, N.I. (1950). Theory of linear operators in Hilbert space, M., 543 c. [in Russian].

2. Samoilenko, A.M. (2002). Averaging of nonlinear oscillating systems of higher approximation with delay. Nonlinear oscillations, V. 5, № 1. P. 77 - 85. [in Russian].

3. Boychuk, I.A. (2010). Nonlinear Noetherian boundary value problem in the critical case. Reports of the National Academy of Sciences of Ukraine, № 3. P. 35 - 40. [in Russian].

4. Iosida, K. (1967). Functional analysis, M .: Mir, - 624 c. [in Russian].

5. Kovalenko, V.F., Kukharchuk, N.M., \& Semenov, Yu.A. (1985). On the theory of diffusion processes generated by the operator. Dep. in UkrNIINTI. Kiev, 802380-Uk 85. [in Russian]. 
6. Krasnoselsky, M.A. (1962). Positive solutions of operator equations. M .: Gos. izd-vo fiz.-mat. litry. 394c. [in Russian].

7. Ladyzhenskaya, O.A., Solonnikov, V.A., \& Uraltseva N.N. (1967). Linear and quasilinear equations of parabolic type. M .: Science. 735 c. [in Russian].

8. Lyons J.L. (1972). Some methods for solving nonlinear boundary value problems. M: Миp, 587 c. [in Russian].

9. Ladyzhenskaya, O.A., \& Uraltseva, N.N. (1973). Linear and quasilinear equations of elliptic type. M .: Наука, 579 c. [in Russian]

10. Oleynik, O.A., \& Samokhin, V.N.(1997). Mathematical methods in the theory of the boundary layer. M .: Fizmatlit, 512 p. [in Russian]

11. Semenov, Yu.A. (1982). Smoothness of generalized solutions of the equation with continuous coefficients. Mat. Sat. T.118 (160), №3 (7). P. 399 - 410. [in Russian]

12. Skripnik, I.V. (1992). A necessary condition for the regularity of a boundary point for a quasilinear parabolic equation. Mat. Sat. T.183, №\%\%. P. 3-22. [in Russian]

13. Barbu, V. (1976). Nonlinear semigroups and differential equations in Banach spaces. Legden: Nordhoff International Publiching, $352 \mathrm{p}$.

14. Benjamini, I., Chavel, I., \& Feldman, E.A. (1996). Heat kernel lower bounds on Riemannian manifolds using the old ideas of Nash. Proc. London Math. Soc., V.72. P. $215-240$.

15. Belluce, L.P., \& Kirk, W.A. (1966). Fixed point theorems for families of contraction mappings. Pacific J. Math., V.18, № 2. P. 213 - 217.

16. Berlyiand, A.G., \& Semenov, Yu. A. (1990). On the $L_{p}$-theory of Schrodinger semigroups. Siberian Math., J. V.31. P. 16 - 26.

17. Boychuk I., Starkova, O., \& Tchujko, S. (2009). Weakly perturbed nonlinear boundary-value problem in critical case. Studies of the University of Žilina. Mathematical Series. October, V. 23, № 1. P. 1-8.

18. Brézis, H., \& Pazy, A. (1970). Semigroups of non-linear contractions on convex sets.J. Func. Anal., V. 6, P. 237-281.

19. Browder, F.E. (1965). Existence of periodic solutions for nonlinear equations of evolution. Proc. Nat. Acad. Sci. USA., V. 53, P. $1100-1103$.

20. Browder, F.E. (1967). Nonlinear equations of evolution type and nonlinear accretive operators in Banach spaces. Bull. Amer. Math. Soc., V.73, P. $867-874$.

21. Crandall, M.G., \& Pazy, A. (1969). Nonlinear semi-groups of contractions and dissipative sets. J. Func. Anal., V. 3, P. $376-418$.

22. Chichurin, A.V. (2003). Integration of Chazy equation with constant coefficients. Nonlinear Oscillations., Vol. 6, № 1. P. 133-143.

23. Chichurin, A.V. (2003). Integration of special linear equations of the second order. Nonlinear Oscillations., Vol. 6, № 2. P. 279-287.

24. David, E.E., \& Evans, W.D. (2004). Hardy operators, functional spaces and embeddings. Berlin: Springer,- 326 p.

25. Fabes, E.B. (1993). Gaussian upper bounds on fundamental solutions of the parabolic equation: the method of Nash in Dirichlet forms. Lectures Notes in Math. Berlin: Springer-Verlag, P. 1 -20 .

26. Goldstein, J. (1985).Semigroups of linear operators and applications. Oxford: Oxford University Press, 245 p.

27. Kasyanov, P., \& Zadoyanchuk, N. (2006). Faedo-Galerkin method for the second-order nonlinear evolution equations with the operators of the Volterra type. International Conference on Differential Equations Dedicated to the 100th Anniversary of Ya.B.Lopatynsky: Book of Abstracts (Lviv, September 12-17, 2006)/Ivan Franko National University of Lviv. - Lviv, Ivan Franko National University of Lviv, P.104-105.

28. Kato, T. (1967). Nonlinear semigroups and evolution equations. J. Math. Soc. Japan., V. 3. P. $375-402$.

29. Kato, T. (1980).Perturbation theory for linear operators. Berlin-Heidelberg-New York: Springer-Verlag, $578 \mathrm{p}$.

30. Kato, T. (1967). Non-linear semigroups and evolution equations. J. Math. Soc. Japan., V. 19. P. $508-520$.

31. Komura, Y. (1969). Differentiability of nonlinear semigroups. J. Math. Soc. Japan., V. 21. P. 
375-402.

32. Komura, Y. (1967). Nonlinear semi-groups in Hilbert space. J. Math. Soc. Japan., V. 19. P. $493-507$.

33. Minty, G. (1962). Monotone (nonlinear) operators in Hilbert space. Duke Math. J., V. 29. P. $341-346$.

34. Minty, G. (1967).On the generalization of a direct method of the calculus of variations. Bull. Amer. Math. Soc., V. 73, №3. P. 315 - 321.

35. Miyadera, I. (1966). On perturbation theory for semi-groups of operators. Tohoku Math. J., V. 18. P. $299-310$.

36. Moser, J. (1960). A new proof of De Giorgi's theorem concerning the regularity problem for elliptic differential equations. Comm. Pure Appl. Math. 13, 457-468.

37. Nagy, B. (1976). Spectral mapping theorems for semigroups of operators. Acta Science Math., V. 38. P. 343-351.

38. Nash, J. (1958). Continuity of solutions of parabolic and elliptic equations. Amer. J. Math., V. 80. P. $931-954$.

39. Naniewicz, Z., \& Panagiotopoulos, P.D. (1995). Mathematical theory hemivariational inequalities and applications. Marcel Dekker, Inc., New York, Basel Hong Kong, 267 p.

40. Nirenberg, L. (1955). Remarks on strongly elliptic partial differential equations. Comm. Pure Appl. Math., V. 8. P. 648-674.

41. Opial, Z. (1967). Weak convergence of the sequences of successive approximants for nonexpansive mappings in Banach spaces. Bull. Amer. Math. Soc., V. 73. P. 591 - 597.

42. Pederson, R.N. (1965). On an inequality of Opial, Beesack and Levinson. Proc. Amer. Math. Soc., V. 16. P.174- 234.

43. Papageorgiou, N.S. (1994). Existence of solutions for the second order evolution inclusion. Journal of applied mathematics and stochastic analysis., Vol.7, № 4. P. 525-535.

44. Papageorgiou, N.S. (2006).Second order nonlinear evolution inclusions: structure of the solution set. Acta math. sinica, English series. Vol. 22 № 1. P. 195-206.

45. Papageorgiou, N.S. (1987). On multivalued evolutions equations and differential inclusions in Banach spaces. Comment. math. unaiv. San. Pauli., Vol. 36. P. 21-39.

46. Yaremenko, M.I. (2008). Second order quisi-linear elliptic equation with matrix of Gilbarg Serrin in $R^{l}$ and nonlinear semi-groups of contraction in $L^{p}$. Proceedings of the conference "Twelfth International Scientific Conference named after Academician M. Kravchuk. May 1517, 2008, Kyiv ". Kyiv, C. 473.

47. Yaremenko, M.I. (2008). Second order quisi-linear elliptic equation with matrix of Gilbarg Serrin in $R^{l}$ and nonlinear semi- groups of contraction in $L^{p}$. «International Conference on problems of decision making under uncertainties (PDMU-2008). May 12-17, 2008. » C.43.

48. Yaremenko, M.I. (2017). The existence of solution of evolution and elliptic equations with singular coefficients. Asian Journal of Mathematics and Computer Research, Vol.: 15, Issue.: 3. pp. 172- 204.

49. Yaremenko, M.I. (2017). Quasi-linear evolution and elliptic equations. Journal of Progressive Research in Mathematics. Vol.11., №3, pp. 1645-1669.

50. Yaremenko, M.I. (2016). Sequence of semigroups of nonlinear operators and their applications to study the Cauchy problem for parabolic equations. Scientific Journal of the ternopil national technical university № 4 (84), pp. 149-160.

Одержано 18.08.2020 